Jeri E. Wieringa

\title{
Mining Eschatology in Seventh-day Adventist Periodicals
}

"Move fast and break things." Once the internal motto of Facebook, this approach to the creation of new technologies has shaped cultural patterns around software and the internet, placing a premium on disruption, rapid development, and novelty. Within the digital humanities, much of the early rhetoric around building and experimentation echoed the "build first, ask questions later" ethos of tech communities. As computational methods have continued to develop, the push to stay on the "cutting edge" of new research methods remains strong, with leading figures focusing on applications of deep learning to the textual and visual data of the humanities. While research at the edges of new technologies continues to expand the questions that can be asked, there are open research questions and opportunities all along the lifecycle of digital humanities projects, including data creation and publishing, algorithm development, and preservation. The digital humanities have developed to a point where it is possible and desirable to take a step back and consider what has been accomplished, identify future directions for digital scholarship, and outline the opportunities for collaboration to further the possibilities for and quality of computational research in the humanities.

As an entry point into these questions, I offer my research for A Gospel of Health and Salvation, a digital dissertation project in religious history, to illustrate the opportunities and limitations around computational research in history. The digitization of primary source materials has opened new opportunities for research in the humanities, increasing access to historical records and making new types of data available for analysis. As computational research in the humanities has developed, however, the types of data needed for robust analysis have also shifted. Rather than being a burden, I argue that these shifts create opportunities for scholarly collaboration between researchers, librarians, and archivists to support the creation, use, and management of the data sets and algorithms needed for computational analysis of the past. The success of such collaborations requires a shift in attitude from an emphasis on rapid adoption to one of slow cultivation where the complex, labor intensive, and careful work of data creation, algorithm development, and infrastructure building that will support the next generation of computational scholarship can thrive. 


\section{Building a Text Mining Project in Religious History}

In A Gospel of Health and Salvation I offer a computational analysis of the periodical literature produced by the Seventh-day Adventist denomination (SDA) between 1849 and 1920. My research is organized around two primary research areas. The first, located within the field of American religious history, explores how beliefs about end-times and constructions of gender interplayed in the development of the religious culture of the SDA during their first seventy years. The transition of the SDA from an emerging religious movement to an established denomination was not a linear progression. Born out of William Miller's teachings regarding end-times and committed to the belief that October 21, 1844 had been correctly identified as the date when the prophecies of the biblical book of Daniel were fulfilled, early Seventh-day Adventists were a people in temporal flux. They understood themselves to be living in the last days while continually wrestling with the continuation of time and the lengthening of their understanding of their temporal position in divine history. This period of instability, both temporally and theologically, led to their development of distinctive beliefs and practices around food, health, and, arguably, gender roles.

The relationship between the end-times beliefs of church members and their shifting cultural ideas about gender are in greater relief in early Seventh-day Adventism because the denomination was under the leadership of Ellen G. White. As a woman prophet and religious leader, White embodied and challenged the complex gender norms of nineteenth-century America. She advocated the sharing of domestic labor between spouses, the wearing of the "American costume," and the need for equitable pay for women denominational workers. She also encouraged women to embrace domestic work as their primary religious calling and was conspicuously silent on questions of women's ordination. The tensions within her writing and her life have made her a challenging figure to understand, particularly in terms of standard accounts of women in radical or emerging religious movements. ${ }^{1}$

1 Scholars who study women in religious movements have frequently used the frameworks of Mary Douglas and Carroll Smith-Rosenberg to explain the prominence of women in leadership during periods of religious revival in terms of the loosening of social structures in the face of economic and social disruption. One of the few scholars to study gender roles in Seventh-day Adventism, Laura Vance, uses this framework, and notes that it is difficult to characterize Ellen White's role in terms of defining gender roles as it is at times progressive and expansive, while at others conservative and restrictive. Mary Douglas, Purity and Danger: An Analysis of 
The second research area I explore in A Gospel of Health and Salvation is methodological, examining the practical and theoretical implications of the application of computational tools to the study of history. Most of the tools used for natural language processing of digital texts, such as named entity recognition and machine learning methods such as topic modeling, were created using texts produced in the twentieth century that consist of technical or academic content. Undertaking computational work as part of historical analysis with digitized sources is still relatively rare and brings with it different challenges and constraints. Through the dissertation, I researched the process of moving from digitized content to historical interpretation, attending to questions of data verification, code reproducibility, and interpretation with complex computational models. In doing so, I raised questions of whether the data and tools currently at hand for computational analysis are sufficient to support robust historical analysis.

To begin a discussion of the future of computational methods in digital history, I will trace my journey from data collection to interpretation of a large collection of the SDA periodical literature. This includes the sourcing and selecting of digital artifacts to work with, the transformation of those into data, the analysis of that data using machine learning, and the interpretation of those results. While somewhat technical at times, these processes constitute a major part of my research in the methodological portions of the dissertation and shape my assessment of the current state and future possibilities for data work in religious studies. They also provide a concrete example of the ways digitized historical artifacts can be used for computational analysis, ways that often go far beyond the initial goals in digitization and publication.

I gathered my data for A Gospel of Health and Salvation from the online archives of the Seventh-day Adventist Church. ${ }^{2}$ One of the advantages of working with religious history is that there are often networks of volunteers interested in new technologies and in sharing materials with broad audiences. For Seventhday Adventists, the digitization of their periodical history began in the 1980s with the volunteer work of lay church members with an "interest in computers."3

Concepts of Pollution and Taboo (Psychology Press, 2002); Carroll Smith-Rosenberg, Disorderly Conduct: Visions of Gender in Victorian America, (Oxford: Oxford University Press, 1986); Laura Lee Vance, Seventh-Day Adventism in Crisis: Gender and Sectarian Change in an Emerging Religion (Champaign, IL: University of Illinois Press, 1999).

2 "Online Archives," Office of Archives, Statistics, and Research of the Seventh-day Adventist Church, accessed May 10, 2018, http://documents.adventistarchives.org/default.aspx.

3 Details about the history of the digital archives are not readily available on the SDA websites. This description is based on an email exchange with the manager of the Adventist Digital Li- 
This work was supported first by the Ellen G. White Estate and then by the Office of Archives, Statistics, and Research (ASTR) of the General Council of the denomination. The digitization efforts of the denomination continue to expand with the creation of the Adventist Digital Library, which seeks to "spread the gospel of Jesus Christ to the world through direct and unlimited access to Adventist historical materials," and aggregates content from a range of SDA organizations, including the General Conference, the White Estate, and many of the Adventist colleges and universities. ${ }^{4}$

The SDA was a prolific denomination during its first seventy years in terms of periodical production. Print served as a central mechanism by which early members, many of whom were geographically dispersed, connected with one another and created a community of believers. ${ }^{5}$ The first publications were produced by Ellen White's husband, James, and as the denomination grew, members established new publishing houses as part of their missionary activities. Starting in the 1860s, subject-focused periodicals began to proliferate, such as the Health Reformer, and with a reorganization of the denomination around 1900, local and regional conferences began to produce their own titles for community news to supplement the large denominational publications. The amount of published materials, much of which has been digitized, poses something of a challenge of abundance for the textual analysis of the literature of the denomination. ${ }^{6}$ While computational methods enable scholars to work with much larger scales of content and in different ways than are possible for human readers alone, it is helpful to focus the collection under evaluation to achieve more interpretable results.

I focused my analysis on materials published within the United States, and specifically on content produced within four main centers of denominational activity: the Great Lakes region; California and the surrounding southwest states; Washington, D.C., and the surrounding mid-Atlantic states; and the states at the southern end of the Mississippi river basin. These four areas mark the shift-

brary. C. Eric Koester, "RE: Inquiry Regarding Data from the Adventist Digital Library,” October 26, 2017.

4 “About," Adventist Digital Library, 2018, https://adventistdigitallibrary.org/about.

5 This process parallels the development of national identity through the creation of an "imagined community" as described by Benedict Anderson. Benedict R. O'G. Anderson, Imagined Communities: Reflections on the Origin and Spread of Nationalism, Rev. and extended ed., 2nd ed. (London; New York: Verso, 1991).

6 Roy Rosenzweig, "Scarcity or Abundance? Preserving the Past in a Digital Era," The American Historical Review 108, no. 3 (June 2003), http://www.historycooperative.org/journals/ahr/108.3/ rosenzweig.html. 
ing centers of power and missionary activity within the denomination, as the denomination moved their headquarters from Michigan to Washington, D.C., and as the center of health reform shifted from Michigan to California as John Harvey Kellogg and the Battle Creek Sanitarium fell from favor. Additionally, apart from regional and college papers, nearly all the major periodicals of the denomination were produced from within these four geographic regions. Using these constraints, I limited my corpus for analysis to the thirty periodical titles produced in these four regions and available as digital files from the ASTR website.

The transformation of the collection of documents into data was made simpler because the periodical scans available through the ASTR had already been converted into machine readable data using OCR (Optical Character Recognition). This enabled me to extract the text, which served as my primary data set, and use machine learning algorithms to identify patterns of language use. My data consists of 13,340 periodicals issues, comprised of 197,943 pages of material, 244,564,660 total words, and 1,537,257 unique words.

This scale of content is best described as "medium" sized data-not so large that it could not be processed by a single reader with enough time, but large enough that it is difficult to interpret without some sort of overview or summary data. To find patterns within my corpus, I used topic modeling, an unsupervised classification algorithm that groups words into an arbitrary number of "topics" based on their co-occurrence within documents. ${ }^{7}$ Unsupervised in this case means that I passed all the documents to the algorithm with no contextual information other than the number of topics to divide the words into and some additional parameters controlling the way the algorithm processed the data. The main alternative to unsupervised is supervised learning, where a researcher identifies pages as containing, say, "domestic" content, "health" content, or "theological" content, and then uses an algorithm to identify the features that distinguish those categories from one another, and then uses that model to predict the category of future materials.

Topic models generate a high-level overview of a corpus of literature, using patterns in word usage to identify different topics or subjects of discourse. Developed initially to address the problem of identifying relevant content within the exponentially-growing universe of scientific literature, the topic model is optimized for problems of information retrieval and summary. Although it works best with the more regular and topically-focused content of academic journals,

7 For an introduction to potential uses of topic modeling in theological libraries, see Micah Saxton, “A Gentle Introduction to Topic Modeling Using Python," Theological Librarianship 11, no. 1 (April 5, 2018): $18-27$. 
it has been used to explore the content of a range of textual artifacts, including novels, poetry, newspapers, and diaries. ${ }^{8}$

MALLET was one of the early libraries released for creating topic models, and has had wide adoption within the digital humanities community because of its (relatively) easy to use interface. As researchers have begun to experiment with topic models for modeling relationships between textual features and variables such as the gender of an author, the time of publication, and other metadata aspects, new and more complex versions of the algorithm have been released. These libraries, such as Structural Topic Model (STM) and Dynamic Topic Model (DTM), factor in these different relationships as part of the model, and provide tools for computing the effect of the different variables on the topic distribution. This enables a statistical calculation of the effect of different variables on structure of the model, such as the effect of new source on the frequency and type of coverage for a topic. ${ }^{9}$

For my dissertation, I used the more basic topic modeling algorithm of MALLET to reduce the complexity of the algorithmic assumptions at play in tracking the discourse of the denomination over time. Modeling the relationship between discourse and time is not straightforward, and as demonstrated by Ben Schmidt, the model of change over time assumed in more complex topic modeling algorithms is one of gradual and continuous change. Such models do not account well for moments of historic rupture or for communities where a cyclical pattern

\footnotetext{
8 Some high-profile projects in the digital humanities that use topic modeling on a range of different types of content include Ted Underwood and Andrew Goldstone's exploration of the archives of the PMLA journal, Lisa Rhody's study of ekphrasis poetry, Robert Nelson's work with the Richmond Daily Dispatch and Sharon Block's work with the Pennsylvania Gazette, and Cameron Blevins's explorations of Martha Ballard's diary. Andrew Goldstone and Underwood, Ted, "What Can Topic Models of PMLA Teach Us About the History of Literary Scholarship?," Journal of Digital Humanities 2, no. 1 (2012), http://journalofdigitalhumanities.org/2-1/whatcan-topic-models-of-pmla-teach-us-by-ted-underwood-and-andrew-goldstone/; Lisa M. Rhody, “Topic Modeling and Figurative Language," Journal of Digital Humanities 2, no. 1 (April 7, 2013), http://journalofdigitalhumanities.org/2-1/topic-modeling-and-figurative-language-by-lisam-rhody/; Robert K. Nelson, "Introduction," Mining the Dispatch, accessed August 17, 2018, http://dsl.richmond.edu/dispatch/pages/home; Sharon Block, "Doing More with Digitization," Common-Place 6, no. 2 (January 2006), http://www.common-place-archives.org/vol-06/no-02/ tales/; Cameron Blevins, “Topic Modeling Martha Ballard's Diary,” Cameron Blevins (blog), April 1, 2010, http://www.cameronblevins.org/posts/topic-modeling-martha-ballards-diary/.

9 For an example of this type of research, see Margaret E. Roberts, Brandon M. Stewart, and Edoardo M. Airoldi, "A Model of Text for Experimentation in the Social Sciences," Journal of the American Statistical Association 111, no. 515 (July 2, 2016): 988-1003, https://doi.org/10. 1080/01621459.2016.1141684.
} 
is operative. ${ }^{10}$ For early Seventh-day Adventists, time was not a static category of experience-time and the experience of temporality were part of what denominational members contested and were striving to understand. As a result, an algorithm with opinions about time muddies the water, so to speak, by imposing an external structure on one of the very aspects that I am looking to explore.

Having gathered an appropriate data set and selected a processing algorithm, my final processing step was to create the topic model. I selected two hundred and fifty as the target number of topics and to improve the quality of the model, limited the documents passed to MALLET to pages with more than three hundred words and error rates under ten percent. Rather than use an existing wordlist, which is the default MALLET process for removing high frequency but low meaning words, I used another topic modeling library, Gensim, to identify those words that occurred in more than sixty percent of documents (high frequency words) and those that occurred in fewer than twenty documents (low frequency words) and used that list as my stopwords list. Using this method, I generated a word list appropriate to the specialized language of the SDA. My final result was a model with some overlap in topics, but with enough specificity to serve as a useful guide to the content of the periodical pages in my study.

Generating a topic model is the first part of the challenge; using and interpreting a topic model within historical analysis is a second and less commonly discussed project. For classification problems, where a researcher uses the model to generate and assign topical categories to different documents, outputs such as the breakdown of topic percentages for each document allow the researcher to identify the most prevalent topics in each document, and use the associated label as a descriptive tag or category. ${ }^{11}$ Topic models also provide an overview

10 Benjamin M. Schmidt, “Words Alone: Dismantling Topic Models in the Humanities,” Journal of Digital Humanities 2, no. 1 (2012), http://journalofdigitalhumanities.org/2-1/words-alone-bybenjamin-m-schmidt/.

11 It is important to note that topic labels are an additional interpretive layer created by the user, but are not part of the topic model output. One common automated labeling strategy is to take the top five words for each topic to serve as the label. This strategy has the advantage of being fast and, with the right content, relatively easy to interpret. For more detailed uses of the topic label, however, it is desirable to move beyond the words clustered together by the model to a label that provides an interpretation of those words and the documents where those topics are prevalent. For example, the top six words of topic \#45 (kingdom shall king daniel great prophecy) and topic \#215 (daniel vision week end dan prophecy) from A Gospel of Health and Salvation are difficult to distinguish with an automatically generated label. However, when interpreted in the context of a larger sample of the topic words and the documents where these topics are prevalent, it becomes apparent that topic \#45 captures documents that strongly feature the figures (the beasts, Nebuchadnezzar's statue, etc.) of the book of Daniel, while topic 
of topic distributions across the entire set of documents, providing a snapshot of the major and minor themes in a corpus. As a form of machine learning, topic models can be used to classify new content, enabling researchers to use a previously generated model to classify previously unseen content.

Researchers in the digital humanities have pursued several different strategies to bridge the computational and abstract data of topic models with the interpretive questions of the humanities. My goal in working with a topic model is not to argue for correlations between different facets of SDA discourse, but to surface broad patterns and identify areas for further research. This use of a computational model fits within the epistemological commitments of the humanities, with its emphasis on complexity, multiple causality, and the idiographic, rather than using the model to argue for correlations between discourse patterns and metadata variables. ${ }^{12}$ My primary areas of interest are within the humanities -finding ways to explore particular aspects of religious culture, to understand how a belief system works and changes, and to consider how those patterns of thought have been built upon to shape the current cultural landscape. These are questions that I believe computational models and methods can help in the exploration of, but as one method among others, including traditional archival research and narrative construction.

Approaching the topic model as an abstraction of general patterns in the periodical literature of the denomination, I focused on those topics that contained language I identified as relating to general concerns about the end times, or eschatological topics. For early Seventh-day Adventist authors, discussions of the end of time tended to rely on illusions and metaphors, particularly using biblical language from Daniel and Revelation, as well as discussions of the significance

\#215 captures documents focused on parsing the meaning of dates in the prophetic books of Daniel and Revelation. While a longer process that requires subject-matter knowledge, this approach results in more meaningful labels that reflect both the words within the topic and the documents described.

12 Tanya Clement provides a useful discussion of the need to explicitly link computational methods to the epistemological frameworks of the humanities as part of the process of connecting such work to the larger intellectual tradition in Tanya Clement, "Where Is Methodology in Digital Humanities?," in Debates in the Digital Humanities, ed. Lauren F. Klein and Matthew K. Gold, 2016 ed. (Minneapolis: University of Minnesota Press, 2016), http://dhdebates.gc. cuny.edu/debates/text/65. This is not to say that one cannot pursue a social-scientific study of traditionally humanities subject areas, such as literary history, where one uses computational methods to statistically model and argue for the relationships between different aspects of one's data. However, this is not the approach I am pursuing with the periodical literature of the SDA, choosing instead to use the computational data for description and as a guide to interpretive research. 
of the dates and periods of times described in the Bible. A second set of topics that I identified were those related to eschatology, including descriptions of the second coming, heaven, and the events of the last days, as well as more theological topics such as conditional immortality and the sanctuary doctrine. ${ }^{13}$ Third, I identified those topics that captured concerns regarding "signs of the times," the purported evidence of prophecy being fulfilled and the threats of growing disorder. Finally, I identified topics related to the separation of church and state, and particularly the Sabbath reform attempts of the late nineteenth century, which greatly concerned Seventh-day Adventist authors as both a challenge to their faith and a sign of the coming end.

Within these topical categories, I identified twenty-four topics generated by the model as linked to discussions around end-times topics. I then aggregated the prevalence of these topics over time, computing the percentage of tokens assigned to the topics in each year, both across the entire corpus and within the main denominational periodicals. (See figures 2 and 3.) This yearly aggregation of topic assignments is an additional layer of abstraction on top of the topic model, one that helps illuminate general patterns. It is important to note that aggregating topics over time can be done in multiple ways, including finding the average prevalence of a topic in each year, measuring the proportion of documents where the topic is dominant, and computing the aggregated percentage of tokens assigned to each topic in a year. Each mode of aggregation provides a different abstraction of the topic model, useful for exploring different questions of the data. The resulting patterns indicate a shift in the language of end-times expectation over time, from more explicit discussions of theology early in the denomination's history to an increased focus on precipitating events later in the period. The charts show spikes in these topics between 1851 and 1862, during the early 1870s, the years around 1890, and the period of World War I.

Seventh-day Adventist theologian Jon Paulien has documented over twenty formal predictions of the second coming throughout the history of Seventh-day Adventism, including in 1844, 1845, 1851, 1884, 1888, 1894, and $1918 .{ }^{14}$ Overall, the model reflects periods of heightened discussion of end-times in the years prior to these formal dates, particularly in the early and later years of this study. The topic model diverges from the predictions documented by Paulien between 1860 and the mid 1880s, where the model indicates additional waves of

13 For more on these theological positions, which are distinctive to the SDA, see Malcolm Bull and Keith Lockhart, Seeking a Sanctuary, 2nd ed. (Bloomington: Indiana University Press, 2007), http://www.jstor.org.mutex.gmu.edu/stable/j.ctt1b349jq, particularly chapters 4 and 5.

14 Jon Paulien, What the Bible Says About the End-Time (Hagerstown, MD: Review \& Herald Publishing Association, 1998), $20-23$. 
Proportion of Words in Full Corpus

Assigned to All End-Times Categories, Aggregated Yearly.

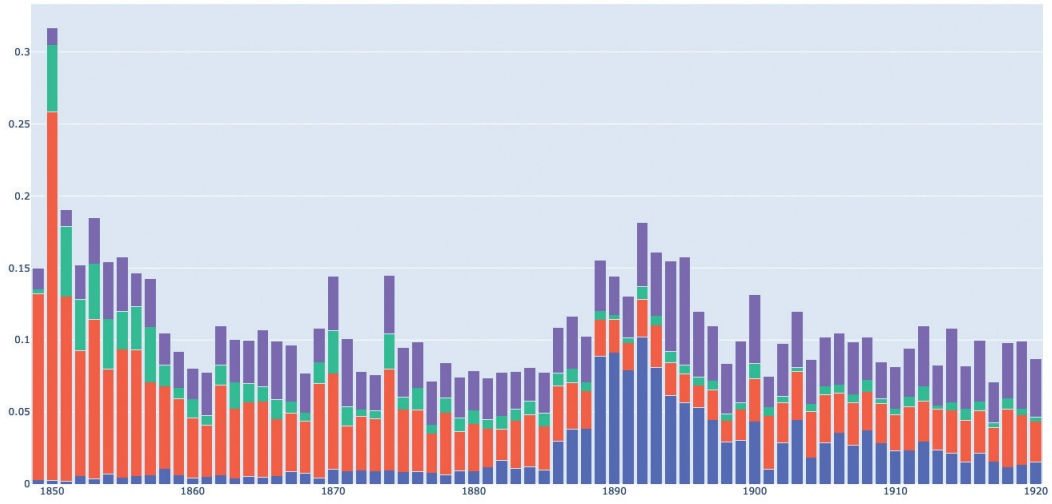

Figure 1: Combined prevalence of end-times topics in all periodical titles, except Youths' Instructor.

Proportion of Words in Denominational Titles

Assigned to All End-Times Categories, Aggregated Yearly.

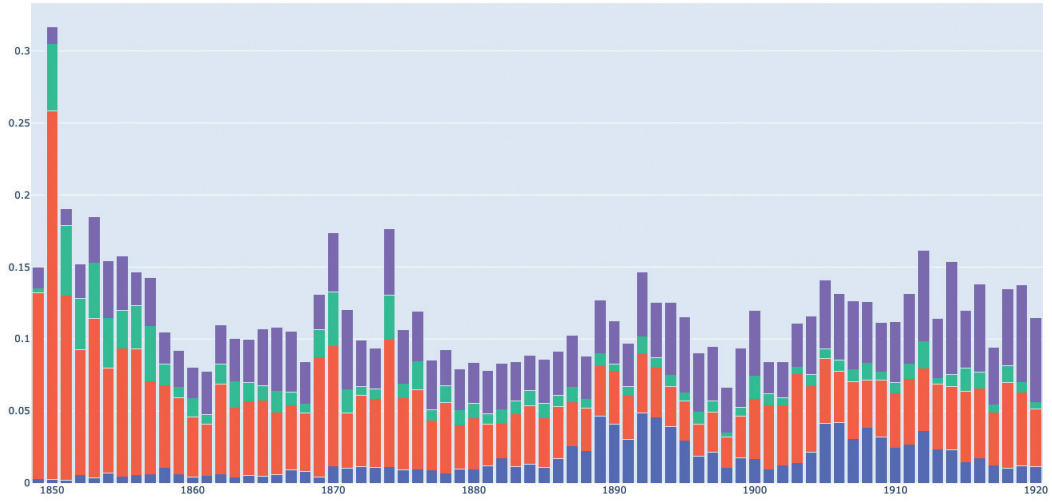

Figure 2: Combined prevalence of end-times topics in five major denominational titles Review and Herald; Signs of the Times; Adventist Review Anniversary Issues; Present Truth and The Adventist Review; and The Church Officers Gazette.

heightened expectation but shows lower than expected levels of interest in endtimes during the early 1880 s. This disconnect between the model and the secondary literature indicates a point for further analysis to better understand the pat- 
terns of language use that the model has captured and to examine how these two seemingly conflicting pieces of information relate to one another.

Using the topic model also allows me to dig deeper into the discourse of denominational members during these cycles of expectation and disappointment. This is where an unsupervised text mining algorithm is particularly useful. A more traditional method of finding such patterns would be to read through a range of periodicals to locate examples of "end times" discourse, and to discuss the patterns seen across the gathered examples. This is a time-intensive process and a selective one. To then examine the co-occurring shifts in discourse surrounding these discussions would require additional surveys through the literature. Topic modeling, as a guide to sources, provides an alternative method for identifying related topics within primary materials. Because I used the topic model to categorize all the text, not just those pages related to end-times discussions, and because I did not set those categories prior to categorization, I can use the model to expand my study beyond the question of when end-times expectation was particularly intense to what other topics were of concern prior to and after these periods of heightened expectation, and what that reveals about the cultural development of the SDA.

Topic modeling, as with all computational analysis in the humanities, provides new forms of evidence and new modes of investigation but does not in and of itself provide answers to the questions of meaning and interpretation that are the primary focus of scholarship in the humanities. As data to be used in the process of meaning-making, the strength of that data is dependent upon the underlying source data, the handling of that data, and the methods used in analysis. My contention is that this relationship between the creation of the model and its use in interpretive work necessitates that the technical work be treated not as background, preprocessing work, but as core to the intellectual activity that is scholarship in fields such as the digital humanities. As such, the work of data creation and processing results in scholarly products that open new areas of research, that ground the interpretive claims of the resulting narratives, and that require their own processes around publishing and preservation.

\section{Current Challenges for Computational Text Analysis in Religious History}

Online archives of digitized material enabled the kind of computational research I performed with the periodical literature of the SDA, but the use of this data is 
not without its challenges. Early digitization projects were primarily focused on increasing access and discoverability of archival materials. To support these goals, early research on the textual data from scanned materials focused on the robustness of keyword searching in the face of OCR errors and on the creation of item-level metadata sufficient for document retrieval. ${ }^{15}$ These goals were a necessary priority in the effort to create and disseminate digital resources. However, computational analysis, such as what I undertook for my dissertation, creates new requirements on digital and digitized data sources, with needs for cleaner textual data and more expansive supporting metadata. By and large, these requirements are not being met with the first-generation work of digitization that is distributed by large scale vendors such as ProQuest and Gale, and through smaller digital archives, such as the Adventist Digital Library. To illustrate these shifting data needs, it is necessary to discuss the less glamorous work I undertook to evaluate and prepare the textual data for analysis, as well as the limitations on the types of analysis possible due to the quality of the data. My goal here is not to criticize current digital resources, but to draw attention to the ways computational analysis changes the requirements for digital resources and point to the possibilities for ongoing collaboration between researchers, archivists, and librarians in meeting these changing data needs.

My first concern with the data from the ASTR, and with any online database, was coverage, or how well the digitized materials reflected the original print materials. Since I am working with periodicals, this included both missing issues within digitized periodical titles, and which of the published titles were available in digital formats. Using the data from the file names, I charted the number of issues per year for each of the thirty titles I had identified. This provided me with an overall view of the corpus, and revealed a few trends that have shaped my analysis. First, while the primary denominational publication, The Review and Herald, has good coverage during nearly every year of my study, smaller periodicals, such as The Youth's Instructor which was written for the children of the denomination, are much less consistently available over the period. (See Figure 3.) In addition, the total number of periodicals expands drastically in the second half of my period, as regional and topical periodicals began to flourish. These material realities of the historical record shape the possibilities for analysis. In the case of computational analysis, because absences and abundances are mag-

15 Patrick Spedding, “'The New Machine': Discovering the Limits of ECCO,” Eighteenth-Century Studies 44, no. 4 (2011): 437-53; Kazem Taghva, Thomas Nartker, and Julie Borsack, "Information Access in the Presence of OCR Errors," in Proceedings of the 1st ACM Workshop on Hardcopy Document Processing, HDP '04 (New York, NY, USA: ACM, 2004), 1-8, https://doi.org/10.1145/ 1031442.1031443. 
nified as the sources are translated into data, it is vital to identify the strengths and weaknesses of the digital corpus, and to use that information in the processing and interpretation of the resulting computational models.

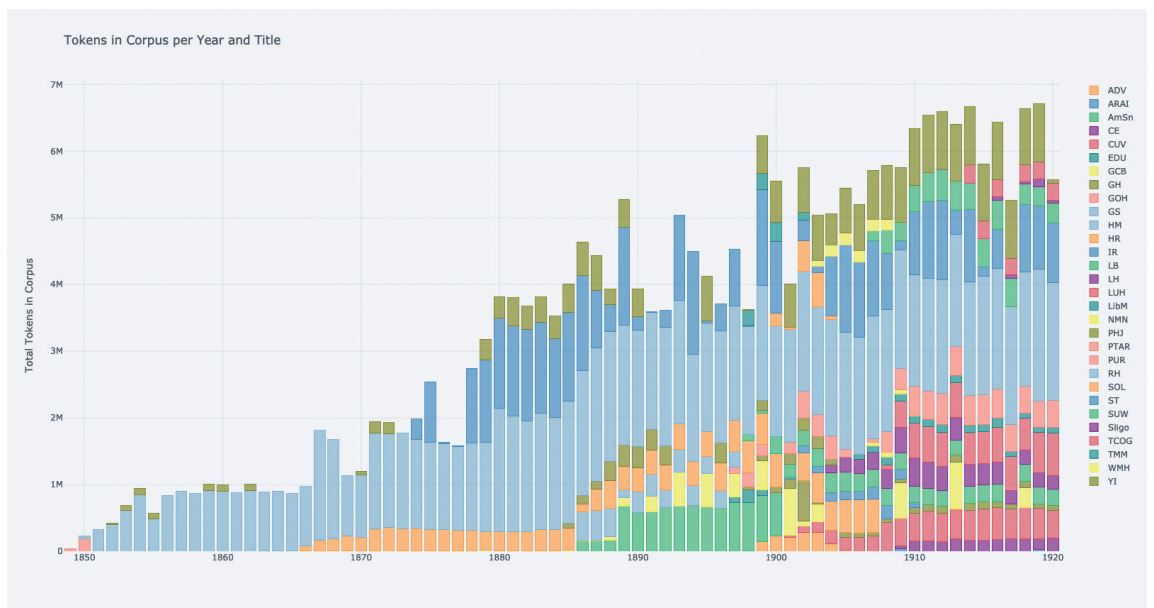

Figure 3: Chart of periodical issues digitized by the SDA and included as part of the textual analysis.

The second challenge in evaluating the coverage of my corpus was to identify the correspondence between the digitized archive and the original publication records of the denomination. For this, I used the yearbooks published by the denomination starting in 1883 to compare the record of periodicals produced by the denomination with those available in the online databases. I found that all the major titles produced by the denomination from my regions of study had a presence in the digital archive and approximately 63 percent of all major and local periodicals titles were available in digital archive. ${ }^{16}$ This breadth of coverage is notably high, with the digital record providing a broad sample of the periodical literature circulating among denomination members. The coverage is also constrained, however, by the interests of the denomination. John Harvey Kellogg's Good Health publication, for example, is included within the digital archive up until 1907, when he was disfellowshiped, even though the publication itself

16 This percentage drops to $55 \%$ if college periodicals are included, which is unsurprising given that student publications tend to be informal and more likely to push against the main denominational positions. 
continued to be published through the 1940s. ${ }^{17}$ This reflection of theological concerns within the digital archive is not surprising, but also constitutes necessary context when interpreting the patterns captured in the model.

As reliance on digital materials expands for both traditional and computational research in the humanities, making available documentation regarding the context of those efforts is an important step to support research in this medium. Similar to the need to document provenance of archival materials, the relationship between digital sources and the archival materials is an important consideration when working with historical sources. There are always gaps in the historical record, and those gaps have generally been enlarged within digital collections. While it is incumbent upon researchers to be aware of and account for archival absences, there are opportunities for collaboration between researchers, librarians, and archivists in creating, releasing, and maintaining records of the known and digitally available resources for analysis.

The second point of concern with the digital artifacts and textual data generated for search and retrieval is data quality. The quality of textual data produced via OCR is a known concern for text-analysis projects. Two recent studies where researchers evaluated the quality of the text data generated from newspaper scans using OCR demonstrate the methods for evaluating the quality of the data. Mapping Texts, which set out to create an interface for the content of the Texas newspapers in the Library of Congress collection, evaluated the textual data by comparing the words in a sample of the extracted text against the Aspell spell-check program. ${ }^{18}$ Even after some basic cleaning of the texts, the team found the word recognition rate to range from $86 \%$ for the titles with the highest quality data to $52 \%$ for the lowest quality titles. ${ }^{19}$ A second study, this one undertaken by a team based at King's College London, took a different approach to ascertaining OCR accuracy, calculating the best accuracy rates achieved from OCR of nineteenth century newspapers in the British Library collection. They did this by comparing samples of the OCR generated text to transcriptions of the newspaper text. On average, this study computed an overall word accuracy of $78 \%$, and a "significant word" accuracy-content words excluding stop words - of

17 There are a few exceptions to the observation: six issues from 1937 and one from 1942 are also available at the Adventist Online Archives. But overall, the publication drops out of the SDA's online record in 1907.

18 Andrew J. Torget et al., "Mapping Texts: Combining Text-Mining and Geo-Visualization to Unlock the Research Potential of Historical Newspapers," Whitepaper (National Endowment for the Humanities), http://mappingtexts.org/index17dc.html?page_id=271, p.15.

19 Torget et al., “Mapping Texts,” p. 23. 
$68.4 \%$ for the $19^{\text {th }}$ Century Newspaper collection. ${ }^{20}$ While OCR is a necessary mechanism when working with scanned objects at scale, these error rates are high enough to raise serious questions about the reliability of this data. ${ }^{21}$

Because many of the PDF files were created by volunteers, there is little documentation as to the OCR processes and expected quality of the textual data for the periodicals of the SDA. A quick read through the text files indicated that, while overall the text appeared readable, there were enough errors to raise questions about the OCR quality across the entire corpus. To evaluate the textual data, I pursued a similar approach to that of the Mapping Texts team, comparing the tokens in the OCRed text to an authoritative wordlist. This entailed creating a wordlist that was appropriate to the language of the denominational periodicals. Standard wordlists are compiled from modern English dictionaries and are appropriately conservative in their inclusion of words. However, they proved insufficient for working with the printed literature of the denomination, which is heavy in language from theology, nineteenth century medicine, and educational theory. Starting from a base wordlist from the SCOWL database, and working through the lists of frequently identified errors, I created a SDA wordlist, which I used to evaluate the tokens that appeared in the text and which served as a baseline to evaluate the success of my error correction measures. ${ }^{22}$ After some cleaning of the texts, the percentage of verified tokens ranged from $98.6 \%$ overall rate for the cleanest periodical title to $92.2 \%$ overall rate for the title with the most errors.

Overall, these error rates are low, particularly when compared to the data reported by the "Mapping Texts" team. This low rate can be attributed in part to

20 The authors note that in their experience a word accuracy above $80 \%$ is best for search and retrieval tasks, a threshold that these results fall beneath. In addition, other studies have shown that more complex textual processes, such as word ranking, are more sensitive to OCR errors than search and retrieval tasks, as errors tend to increase the number of identified tokens, thereby affecting the measure prevalence of individual words. See Kazem Taghva, Thomas Nartker, and Julie Borsack, "Information Access in the Presence of OCR Errors," in Proceedings of the 1st ACM Workshop on Hardcopy Document Processing, HDP '04 (New York: ACM, 2004), 1-8, https://doi.org/10.1145/1031442.1031443, p. 2.

21 Ongoing work, such as the recently funded "History and Multilingual OCR" study at Northeastern University, are beginning to systematically tackle these questions surrounding OCR data for textual analysis. https://ocr.northeastern.edu/

22 SCOWL, or Spell Check Oriented Wordlists, provides numerous wordlists divided into different categories and sizes for use in creating spell check applications. The final wordlist for A Gospel of Health and Salvation included the base SCOWL dictionary, words from the King James Bible taken from Project Gutenberg, US city names, and a list of SDA people and places which I had compiled from the SDA yearbooks. Kevin Atkinson, Spell Checking Oriented Word Lists (SCOWL), version 2016.11.20, http://app.aspell.net/create. 
the use of a comprehensive dictionary against which to compare the text. In addition, it is important to note that because I am evaluating the text against known words rather than the original text, this method does not capture a range of OCR errors. Some examples of the types of errors this approach is blind to include "errors" in character recognition that create verified words, marks correctly captured spelling errors in the original as OCR errors, and errors in layout recognition, which is one of the largest problems in the OCR transcriptions. However, the statistics provide a measure of the percentage of "real word" tokens in the periodical texts and serve as a useful proxy for the quality of the OCR.

After evaluating and cleaning the text, and comparing the results to the original PDF documents, I determined that the text was a good proxy for the original scans, but also that the errors in layout recognition left the text as more of a "bag of words" representation of the periodical pages rather than a digital re-creation of the original print. This quality of the textual data shaped the types of analysis that were most effective. While processes such as search and retrieval, which rely on word recognition, have been found to be generally robust in the face of OCR errors, analysis that requires more complex weighting of words, such as computing the term frequency-inverse document frequency (TF-IDF), are more sensitive to OCR errors that skew the total number of tokens in the documents. ${ }^{23}$ Additionally, analysis that relies on word order, such as part-of-speech tagging and named entity recognition, was unreliable due to the prevalent errors in the textual data.

This labor of evaluating and remediating the text is part of the hidden cost of computational analysis in the humanities. The scale of work that I undertook was necessary because the quality of the textual data influenced the types of analysis the data could reliably support. For the ASTR, the primary goal for the digitized text was to increase discoverability and access of the primary source materials, with the text layer providing added convenience rather than serving as the main output. Other digitized collections have focused on the textual layer as the primary product, such as the digitization of the Richmond Daily Dispatch. ${ }^{24}$ The process of creating clean textual data, however, is labor-inten-

23 Kazem Taghva, Thomas Nartker, and Julie Borsack, "Information Access in the Presence of OCR Errors," in Proceedings of the 1st ACM Workshop on Hardcopy Document Processing, HDP '04 (New York: ACM, 2004), 1-8, https://doi.org/10.1145/1031442.1031443, p. 2

24 The vendor specifications for the digitization and rekeying of the Dispatch scans provides a clear example of the amount of labor involved in producing clean textual data from historical artefacts. See University of Richmond Libraries, "Background Information | Project Information,” Richmond Daily Dispatch, accessed August 17, 2018, http://dlxs.richmond.edu/d/ddr/ 
sive, expensive, and offers few rewards within the current academic structure for the researchers, librarians, and archivists who undertake it. ${ }^{25}$ At the same time, it is this carefully created textual data that is needed as computational text analysis moves from a mode of exploration to a robust mode of analysis within the humanities.

\section{Towards Future Computational Research in the Study of Religion}

The data made available by the SDA is an excellent example of early digitization work that can support computational analysis, and the abundance of digital content related to the history of the SDA is envy-worthy for historians looking to do computational analysis. While the Library of Congress has supported the digitization of newspapers and library vendors make some of their digitized content available for text analysis, these sources start with those materials that appeal to the broadest possible audiences. ${ }^{26}$ For research in the history of religion, by contrast, the availability of digital sources is generally dependent upon the efforts and resources of individual denominations and the work of smaller theological libraries and archives. Having access to an abundance of digital materials, such as those published by the ASTR, makes possible computational analysis with historical data.

However, as I have shown, even with this abundance of digital data there is much work involved in constructing digital studies of religion. Additionally, there are numerous opportunities for collaboration in the creation of data, algorithms, and methods for publication, dissemination, and preservation of complex digital humanities artifacts. Early work in the digitization of archival collections has primarily focused on problems of access and information retrieval-of helping raise awareness of the contents of a collection and enabling search access within and across collections. This work has made it possible for individual

proinfo/bginfo.html and specifically, Andrew Rouner, "Vendor Specifications for Digitization and Rekeying” (Richmond: University of Richmond, 2005), http://dlxs.richmond.edu/d/ddr/ docs/info/vendorspec.pdf.

25 The work of editing and publishing textual data is not always without its recognition and rewards, as the ongoing success of projects such as the Women Writers Project attest. "About the WWP,” Women Writers Project, accessed August 18, 2018, https://www.wwp.northeastern. edu/.

26 Library of Congress, “About Chronicling America," Chronicling America, accessed August 18, 2018, https://chroniclingamerica.loc.gov/about/. 
researchers such as myself to begin to undertake projects based in computational analysis.

In closing this chapter, I would like to draw on my dissertation experiences to suggest additional areas of research and scholarship that will further the possibilities for and the quality of computational research in the study of religion. First, as my work preparing the textual data from the SDA periodicals indicates, there is a need for increased attention to the creation and dissemination of textual data optimized for computational analysis. This is not to say that all digitization projects should require the detailed XML tagging undertaken in projects such as the Richmond Daily Dispatch. While detailed tagging may open additional options for analysis, the more basic need is for the creation of clean data that accurately captures the text of the source documents. The existence of such textual data, while labor intensive to create, greatly improves computational work and makes possible additional research in algorithm creation and analysis. ${ }^{27}$ Such data would support the expansion and maturation of computational analysis with historic sources, and would also increase opportunities for the development of subject-specific tools to expand the options for natural language processing with the specialized language of religious literature.

A second area for further scholarly work is in the creation and dissemination of subject-specific datasets for the study of religion. Well-structured and documented data sets are themselves works of interpretation and analysis, and are scholarly products that would benefit from the collaboration of researchers, librarians, and archivists. Such datasets could include membership roles, editors and locations for publications, the location of houses of worship and other institutions, lists of authorized publications, and the like. As such data increases, the opportunities for surfacing patterns within and across religious groups also expand, but each such dataset represents large commitments of time to reliably produce. Similar to edited volumes, the creation of datasets for historical analysis should be considered an act of scholarship, and one that is done collaboratively.

The third and final area for further scholarship which I wish to note is the need to study and adapt machine learning algorithms and other computational tools for use with historical content and humanities questions. Standard natural language processing tasks, such as named entity recognition, work best when

27 Clean here does not mean "normalized" or corrected. The decision of how to address errors within the original, such as typographical errors or misspellings, depends on the research questions at hand and so should be determined by the individual researcher. For commentary of the trouble of normalized text, see Katie Rawson and Trevor Muñoz, "Against Cleaning," Curating Menus, July 6, 2016, http://curatingmenus.org/articles/against-cleaning/. 
trained on data that is similar to the data one is looking to process. The standard models for part-of-speech tagging and named entity recognition, however, have been trained on primarily twentieth century and news-related content. Studies evaluating their effectiveness with "out of domain” content, which constitutes most of what is used within the humanities, show a marked decline in accura$\mathrm{cy}^{28}$ The development of new algorithms itself is dependent upon the availability of significant amounts of clean textual data. Again, all of this work is labor intensive and interpretive, but vital for there to be a robust "digital humanities" beyond experimentation with new technologies.

The digital humanities have been accused of being just another manifestation of the commercialization and commodification of education and the humanities, and while I generally disagree with that assessment, there are dangers of embracing the "move fast and break things" attitudes of the technology sphere. The areas of work I am proposing here are labor intensive, complex, and require care and imagination, and they are less attractive as they require revisiting ground that is known. Rather than finding the "next new thing" that will illuminate patterns, I am proposing slowing down, clarifying methodology so that the scholarly contributions are understandable, and doing the necessary work of modifying and "refactoring" existing methods to work well with the unique content of religious studies.

The change in emphasis from quick adoption to long term cultivation is also why I see these areas as places where collaborations between researchers, librarians, and archivists can thrive. Datasets are formed to answer questions. There must be research needs behind the creation of text, data, and computational models. At the same time, librarians and archivists have the expertise in data management and preservation, the infrastructure to support and preserve these artifacts as scholarly outputs, and the field awareness needed to make such work viable and useful beyond individual projects. Where the digital humanities are at their most productive is in encouraging collaboration and offering alternatives to existing academic hierarchies. My work for my dissertation highlights that there is much research yet to do to enable robust applications of computational methods to the study of religion.

28 David Bamman, "Natural Language Processing for the Long Tail," 2017, http://people.is chool.berkeley.edu/ dbamman/pubs/pdf/dh2017.pdf. 
